\title{
Coadministration of Divalent Cation Mineral Supplements Causes cART Virologic Failure and HIV Mutagenesis: A Case Study
}

\section{Thomas M Forst and Warren L Dinges*}

\author{
Seattle Infectious Disease Clinic, Seattle, USA
}

\begin{abstract}
Integrase strand transfer inhibitors (INSTIs) are an important component of modern combined antiretroviral therapy (cART). The mechanism of inhibition for these antivirals is dependent on magnesium ion binding during attempted HIV1 replication. Exogenous supplementation of magnesium or other divalent cation minerals may interfere with successful inhibition of HIV replication. This interference has been established in vitro. An additional competitive mechanism evidenced by decreased INSTI bioavailability when coadministered with cations has been observed in dosing studies previously. However, neither mechanism has demonstrated clinical failure of cART. We describe two patients who were taking divalent cation mineral supplements concomitantly with INSTI-cART. Patient 1 developed HIV viremia of $>$ 100,000 copies/mL and significant mutations necessitating a change in cART. Patient 2 developed mild viremia which resolved with removal of the mineral supplement without mutations. In both cases the coadministration of divalent cation minerals led to viremia, and ultimately resulted in cART failure with mutagenesis in one.
\end{abstract}

Keywords

Antiretroviral therapy, Cation, Integrase inhibitor

\section{Introduction}

Patients infected with HIV-1 co-presenting with anemia or gastroesophageal reflux disease (GERD) will frequently turn to dietary mineral supplements or magnesium/calcium-based antacids to alleviate their symptoms. An aging HIV-infected population may also seek to supplement their dietary calcium intake. These may pose dangers to patients whose HIV regimens include integrase strand transfer inhibitors (INSTIs).

Integrase inhibitors are a class of antiretrovirals whose mechanism of action requires divalent cations facilitating a complex between the drug and HIV integrase, with either $\mathrm{Mg}^{2+}$ or $\mathrm{Mn}^{2+}$ being effective both in vivo and in vitro [1]. Examples of current FDA-approved INSTIs include raltegravir, dolutegravir, elvitegravir, with the newest being bictegravir. Previous studies have indicated concurrent administration of antacids and multivitamins containing divalent cation compounds with the INSTI dolutegravir results in a decreased single-dose exposure by $74 \%$ and $33 \%$ respectively, indicating possible chelation in the gastrointestinal tract between the metal cation and the INSTI [2].

\section{Case Presentation}

Here we report two patient cases whose cART regimen included the INSTI elvitegravir in which co-administration of metal cation containing products resulted in an increased HIV viral load. Adherence was self-reported and confirmed by pharmacy refill data. VL was assessed using a commercial clinical laboratory quantitative real time RNA PCR (Roche Diagnostics, Indianapolis, USA), and the co-administration of metal cation containing products was discovered retrospectively. HIV mutations were assessed using GenoSure Prime and GenoSure Archive (Monogram Biosciences, San Diego, USA). Both patients' VLs were decreasing or steady in response to

*Corresponding author: Warren L Dinges, Seattle Infectious Disease Clinic, 509 Olive Way, Suite 752, Seattle, WA, 98101, USA

Accepted: September 05, 2018: Published online: September 07, 2018

Citation: Forst TM, Dinges WL (2018) Coadministration of Divalent Cation Mineral Supplements Causes cART Virologic Failure and HIV Mutagenesis: A Case Study. Clin J HIV AIDS 2(1):26-27

Copyright: (c) 2018 Forst TM, et al. This is an open-access article distributed under the terms of the Creative Commons Attribution License, which permits unrestricted use, distribution, and reproduction in any medium, provided the original author and source are credited. 
Citation: Forst TM, Dinges WL (2018) Coadministration of Divalent Cation Mineral Supplements Causes cART Virologic Failure and HIV Mutagenesis: A Case Study. Clin J HIV AIDS 2(1):26-27

cART but increased with metal cation dosing.

\section{Patient 1}

Patient 1, a 42-year-old man, presented with a new diagnosis of HIV with AIDS wasting with a nadir CD4 count of 84 cells/microL and a HIV VL of 905,200 copies $/ \mathrm{mL}$. At presentation, he was also found to be anemic with a hemoglobin of 11.7 and hematocrit of 36.2. The treatment naive Patient 1 was initiated on INSTI-cART on the day of presentation with elvitegravir/cobicistat/ tenofovir alafenamide/emtricitabine. A multivitamin was also recommended and initiated by the patient. At his first follow-up visit his CD4 had climbed to 182 and his VL had fallen to 30,380. Iron studies were normal except elevated serum ferritin $853 \mathrm{ng} / \mathrm{dL}$. Iron supplementation was recommended in addition to the daily multivitamin. At his next follow-up visit his HIV VL increased from 30,380 copies/mL to 126,570 copies/mL over a period of 18 weeks. The patient reported complete medication adherence, which was confirmed through pharmacy refill data. The patient was called back for drug resistance testing which demonstrated a new mutation which conveyed resistance to one of his nucleoside reverse transcriptase inhibitors (M184V) and INSTIs (N155H). It was discovered retrospectively that the patient was co-administering cART with iron supplements. The patient's cART regimen was switched to dolutegravir/cobicistat/darunavir/tenofovir alafenamide/emtricitabine, iron supplementation was ceased, and viral load reduced to 30 copies $/ \mathrm{mL}$ in 4 weeks. His anemia and AIDS wasting resolved with normal diet after HIV-suppression.

\section{Patient 2}

Patient 2, a 49-year-old man, was well established in HIV-care on suppressive cART. He was initially diagnosed with HIV-infection at 29 years of age and his sequential regimens included efavirenz, lamivudine, tenofovir disoproxil, emtricitabine with the last being elvitegravir/cobicistat/emtricitabine/tenofovir disoproxil fumarate (DF) of greater than 4 years duration. With the new FDA-approval of elvitegravir/cobicistat/emtricitabine/tenofovir alafenamide, the patient switched from elvitegravir/cobicistat/emtricitabine/tenofovir DF to elvitegravir/cobicistat/emtricitabine/tenofovir alafenamide in hopes of avoiding potential bone mineral density and renal side-effects. At post-switch follow-up, the pa- tient presented with a low but detectable VL of 60 . The VL was repeated and sent for drug resistance testing. With confirmation of persistent viremia, an extended conversation was had with the patient. HIV genotyping via GenoSure Prime was not successful due to the low VL. Patient 2 reported recent uses of calcium carbonate (Tums, GlaxoSmithKline, Philadelphia, USA) of unknown dosage to manage GERD symptoms. For Patient 2 , calcium carbonate antacids were replaced with pantoprazole $40 \mathrm{mg}$ daily, a proton pump inhibitor. Subsequent VL testing showed a return to HIV suppression without a regimen change. His GERD symptoms were well managed with the scheduled PPI. In summary, with marked calcium carbonate antacid dosing, the patient's $\mathrm{VL}$ increased from $<20$ copies $/ \mathrm{mL}$ to 90 copies $/ \mathrm{mL}$ over 34 weeks, and mutations assayed via GenoSure Archive did not convey drug resistance.

\section{Discussion}

Previous studies have demonstrated decreased bioavailable INSTI upon co-administration of metal cation containing mineral supplements. However, there is limited data on clinical failure and resultant drug resistance. These two individuals combined cation mineral supplements with INSTI-cART leading to increased HIV viremia and drug resistance in one. While prior evidence supports a decrease in bioavailability of dolutegravir when administered with mineral supplements, there is a lack of evidence for other INSTIs available in once daily cART options. However, all INSTIs requiring magnesium or manganese binding should have a similar problem with exogenous cation minerals. Due to the common usage of these supplements, their interaction with cART including INSTIs, and their potential to induce virologic failure, the use of these supplements must be regularly assessed as part of HIV treatment.

\section{Conflicts of Interest}

WLD has received advisory board, speaker's bureau, and research compensation from Gilead Sciences, Inc.

\section{References}

1. Quashie PK, Sloan RD, Wainberg MA (2012) Novel therapeutic strategies targeting HIV integrase. BMC Med 10: 34.

2. Song I, Borland J, Arya N, et al. (2015) Pharmacokinetics of dolutegravir when administered with mineral supplements in healthy adult subjects. J Clin Pharmacol 55: 490-496. 